\title{
Determination of proper motions and membership of the open star cluster NGC $2548^{\star}$
}

\author{
Z. Y. Wu ${ }^{1,2,3}$, K. P. Tian ${ }^{1,2,3}$, L. Balaguer-Núnez ${ }^{5,1}$, C. Jordi ${ }^{5,6}$, J. L. Zhao ${ }^{4,1,2,3}$, and J. Guibert ${ }^{7}$ \\ 1 Shanghai Astronomical Observatory, CAS Shanghai 200030, PR China \\ 2 Chinese National Observatories, CAS, PR China \\ 3 Joint Lab of Optical Astronomy, CAS, PR China \\ 4 CCAST (WORLD LABORATORY) PO Box 8730, Beijing 100080, PR China \\ 5 Departament d'Astronomia i Mateorologia, Universitat de Barcelona, Avda. Diagonal 647, \\ 08028 Barcelona, Spain \\ 6 Institut d'Estudis Espacials de Catalunya IEEC, Edif. Nexus Gran Capità 24, 08034 Barcelona, Spain \\ 7 Centre d'Analyse des Images, Observatoire de Paris, Bâtiment Perrault, 77 Av. Denfert-Rochereau, \\ 75014 Paris, France
}

Received 7 May 2001 / Accepted 18 October 2001

\begin{abstract}
Absolute proper motions, their corresponding errors and membership probabilities of 501 stars in the intermediate-age open cluster NGC 2548 region are determined from MAMA** measurements of 10 photographic plates. The plates have the maximum epoch difference of 82 years and they were taken with the double astrograph at Zô-Sè station of Shanghai Observatory, which has an aperture of $40 \mathrm{~cm}$ and a plate scale of $30^{\prime \prime} \mathrm{mm}^{-1}$. The

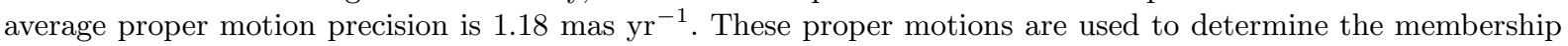
probabilities of stars in the region. The number of stars with membership probabilities higher than 0.7 is 165 .
\end{abstract}

Key words. galaxy: open clusters and associations: individual: NGC 2548 - astrometry - galaxy: kinematics and dynamics

\section{Introduction}

The open cluster NGC 2548, also known as M 48, in Hydra $\left(\alpha_{2000}=8^{\mathrm{h}} 13^{\mathrm{m}} 48^{\mathrm{s}}, \delta_{2000}=-5^{\circ} 48^{\prime}\right)$ seems to be an intermediate-age open cluster with an estimated distance of 630 pc (Pesch 1961) or 530 pc (Clariá 1985). It has not been the object of any recent complete astrometric or photometric study (Ebbinghausen 1939; Li 1954), in spite of being an extended object with an apparent diameter of $30^{\prime}$ (Trumpler 1930) or even $54^{\prime}$ (Collinder 1931) and brilliant enough to be in the Messier list (s.XVIII) with number 48 (Messier 1850). It was even considered inexistent for several years owing to the fact that Messier mistook its real coordinates owing to a change of sign in

\footnotetext{
Send offprint requests to: L. Balaguer-Núñez,

e-mail: Lola.Balaguer@am.ub.es

* Table 5 is only available in electronic form at the CDS via anonymous ftp to cdsarc.u-strasbg.fr (130.79.128.5) or via

http://cdsweb.u-strasbg.fr/cgi-bin/qcat?J/A+A/381/464, and by e-mail request to Lola.Balaguer@am.ub.es

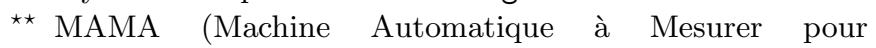
l'Astronomie, http://dsmama.obspm.fr) is developed and operated by INSU/CNRS and Observatoire de Paris.
}

its relative position with a final difference in declination of $5^{\circ}$.

There is no feasible estimation of its age but it seems to be an intermediate-age open cluster, around $\log t=8.5$ (Lyngå 1987), with a slightly poorer CN abundance than the giants of the Hyades but significantly richer than the K giants of the solar neighborhood (Clariá 1985; Twarog et al. 1997).

Proper motions of bright stars in this region were published by Ebbinghausen (1939) with four pairs of plates of only a maximum epoch difference of 28 years. Li (1954) published positions and proper motions in the field of NGC 2548 based on three plates taken with the $40 \mathrm{~cm}$ astrograph at Shanghai Zǒ-Sè station with a maximum epoch difference of only 14 years.

In this paper we determine, for the first time, precise absolute proper motions of 501 stars within a $1.6 \times 1.6$ area in the NGC 2548 region, from automatic MAMA measurements of 10 plates, five of which are newly taken. The estimated membership probabilities lead us to a complete astrometric study of the cluster area. Section 2 describes the plate material and its measurement as well as the proper motion reduction procedure and results with comparisons with the Hipparcos and Tycho-2 catalogues. 
Section 3 accounts for the membership determination. Section 4 is devoted to the analysis of results. Finally, a summary is presented in Sect. 5 .

\section{Plate measurement and proper motion reduction}

\subsection{Plate material and measurements}

Ten plates of the NGC 2548 region were available. They were taken with the double astrograph at the Zô-Sè station of Shanghai Observatory. This telescope, built by Gaultier in Paris at the beginning of the last century, has an aperture of $40 \mathrm{~cm}$, a focal length of $6.9 \mathrm{~m}$ and hence a plate scale of $30^{\prime \prime} \mathrm{mm}^{-1}$. The size of the old plates is $24 \mathrm{~cm}$ by $30 \mathrm{~cm}$, or $2.0 \times 2.5$, and that of the new ones is $20 \mathrm{~cm}$ by $20 \mathrm{~cm}$, or $1.65 \times 1.65$.

The oldest plate was taken in 1916, and the newest ones in 1998. Relevant information on these plates is shown in Table 1. The hour angles are not provided in Table 1 because the starting exposure time of the old plates was not recorded.

All plates were measured at the Centre d'Analyse des Images at the Observatoire de Paris, using the high precision microdensitometer "Machine Automatique à Mesurer pour l'Astronomie" (MAMA). This device has a superb optical and mechanical performance (Guibert et al. 1990). It uses a quartz-iodine illuminating source, whose transmitted light is detected by a reticon, 1024 pixels large, with a pixel size of $10 \mu \mathrm{m}$, and the absolute accuracy of the measurements is $0.6 \mu \mathrm{m}$ (Soubiran 1992). After the plates were scanned, the resulting images were stored in a grid of $19 \times 19$ sub-images for each plate. Once every plate was digitized, we identified all point sources in these 361 frames. The source extraction was performed on each frame using SExtractor (Bertin \& Arnouts 1996), a software dedicated to the automatic analysis of astronomical images using a multi-threshold algorithm allowing good object deblending. To improve the accuracy of the measurements, we chose to retain only rather bright objects with a signal-to-noise ratio of at least 12 .

During the scanning, MAMA includes in the catalogue not only real images, but also the plate grid, emulsion flaws, plate annotations and scratches. A first step is required to reject spurious detections, most of which can be recognized with analysis of the object shape, by removing the plate grid, annotations and big scratches by visual comparison with the original plates. The shape parameters given by SExtractor can be used to clarify the remaining stars but the most direct way to reject spurious detection is by comparison between plates, searching each object from one plate to the other, and retaining only the paired data. There is a total of 596 stars measured with a limiting magnitude $B_{\mathrm{T}}$ around 14 . This limiting magnitude was roughly estimated from the stars in common with Tycho-2 catalogue.

The detection of 182 PPM and ACT stars on the whole plate allowed us to determine the star positions in a common system. Thus for each scanned plate, we obtained a preliminary astrometric catalogue to perform initial cross-identification between plates.

\subsection{Proper motions}

The absolute proper motions for 596 stars in the region of NGC 2548 were reduced on the basis of the MAMA measurements following the central overlapping procedure (Russel 1976; Wang et al. 1995, 1996, 2000). This method determines the plate-to-plate transformation parameters, the star motions and their errors simultaneously. Stellar positions and absolute proper motions were reduced from a catalogue used as the original data for the first iteration. As initial catalogue, 265 stars from the Tycho-2 catalogue at epoch J2000 (Høg et al. 2000) were chosen on the basis of the results of the PPM and ACT astrometric catalogue given by MAMA. To select the best plate constant model, we used Eichhorn \& Williams' criterion (Eichhorn \& Williams 1963; Wang et al. 1982) and obtained a model with six linear constants on coordinates, a magnitude and a coma term, and a magnitude distortion term. Magnitudes used were the instrumental magnitudes. All the proper motions are constrained by having at least one measurement from the modern epoch plates, i.e. taken in 1998. The whole process is iterated until the resulting proper motions converge. We iterate the process until mean differences in position are smaller than 1.1 mas, the rms smaller than 3.6 mas and the differences in proper motion below 0.1 mas yr$^{-1}$, yielding a final outcome of 501 stars.

Table 2 shows mean precisions of final proper motions for stars in the NGC 2548 region detected on different numbers of measured plates (greater than 2). The units of the proper motions and their precisions all along are mas $\mathrm{yr}^{-1}$. The precision of the final proper motions strongly depends on the number of plates. Figure 1 gives the number of stars for which various numbers of plates are available. More than $90 \%$ of proper motions were obtained from at least 5 plates.

The rms errors on proper motions for more than $90 \%$ of stars are $\epsilon_{\mu_{\alpha} \cos \delta}=0.92 \operatorname{mas~yr}^{-1}, \epsilon_{\mu_{\delta}}=0.68 \operatorname{mas~yr}^{-1}$ and $\epsilon_{\mu}=1.18 \operatorname{masyr}^{-1}$, where $\epsilon_{\mu}=\sqrt{\epsilon_{\mu_{\alpha} \cos \delta}^{2}+\epsilon_{\mu_{\delta}}^{2}}$. In the most precise case, the rms errors are 0.77 mas yr$^{-1}$ for stars with 10 plates ( $40 \%$ of stars).

Figure 2 shows the distribution of rms proper motion errors with the number of stars: $N$ versus $\epsilon_{\mu_{\alpha} \cos \delta}, \epsilon_{\mu_{\delta}}$ and $\epsilon_{\mu}$. Thus the precisions of the proper motions of stars in the region of NGC 2548 obtained in this study are relatively high, thanks to the quality of the stellar images taken with the $40 \mathrm{~cm}$ double astrograph and the excellent positioning behavior of the MAMA scanning machine.

Figure 3 gives $\mu_{\alpha} \cos \delta, \mu_{\delta}$ and their errors with respect to instrumental magnitude.

Our absolute proper motions and their errors are compared with those of Tycho-2 catalogue in Fig. 4. We would like to highlight the precision of the proper 
Table 1. Plate material.

\begin{tabular}{cccccc}
\hline $\begin{array}{c}\text { Plate } \\
\text { Id. }\end{array}$ & $\begin{array}{c}\text { Epoch } \\
(1900+)\end{array}$ & $\begin{array}{c}\text { Exp.Time } \\
\text { min }\end{array}$ & $\begin{array}{c}\text { Plate center } \\
\left(\alpha_{\text {J2000 }} \delta_{\text {J2000 }}\right)\end{array}$ & $\begin{array}{c}\text { Plate size } \\
\text { cm }\end{array}$ & $\begin{array}{c}\text { No. of } \\
\text { stars }\end{array}$ \\
\hline CL422 & 16.03 .24 & 90 & $8^{\mathrm{h}} \cdot 2283-5.779$ & $24 \times 30$ & 450 \\
CL534 & 30.03 .21 & 90 & $8^{\mathrm{h}} \cdot 2286-5.775$ & $24 \times 30$ & 556 \\
CL535 & 30.03 .28 & 90 & $8^{\mathrm{h}} \cdot 2291-5.767$ & $24 \times 30$ & 566 \\
CL56006 & 56.03 .14 & 60 & $8^{\mathrm{h}} \cdot 2288-5.779$ & $24 \times 30$ & 577 \\
CL56007 & 56.03 .16 & 60 & $8^{\mathrm{h}} \cdot 2372-5.779$ & $24 \times 30$ & 558 \\
CL98004 & 98.04 .03 & 30 & $8^{\mathrm{h}} \cdot 2447-5.958$ & $24 \times 30$ & 548 \\
CL98047 & 98.12 .16 & 30 & $8^{\mathrm{h}} \cdot 2227-5.796$ & $20 \times 20$ & 268 \\
CL98Tian & 98.12 .25 & 30 & $8^{\mathrm{h}} \cdot 2255-5.804$ & $20 \times 20$ & 268 \\
CL98Chen & 98.12 .25 & 30 & $8^{\mathrm{h}} .2255-5.804$ & $20 \times 20$ & 298 \\
CL98Gu & 98.12 .16 & 30 & $8^{\mathrm{h}} \cdot 2227-5.796$ & $20 \times 20$ & 432 \\
\hline
\end{tabular}

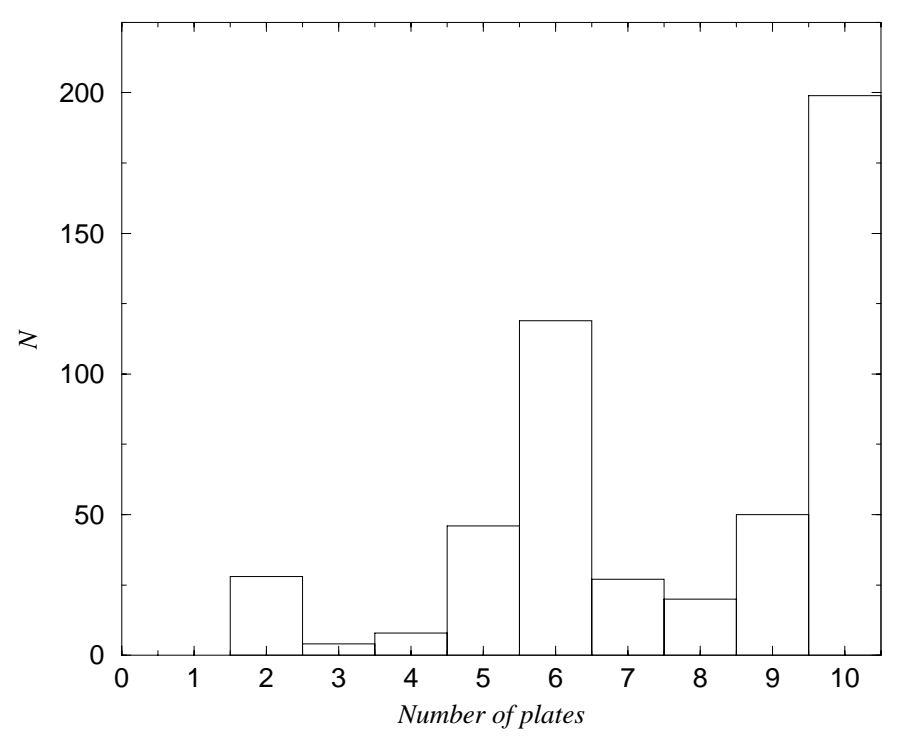

Fig. 1. The number of stars $v s$ the number of available plates.

motions derived in this paper. Mean differences in the sense ours minus Tycho-2 are $-0.123(\sigma=2.112)$ and $-0.203(\sigma=2.158)$ in $\mu_{\alpha} \cos \delta$ and $\mu_{\delta}$, respectively. No apparent systematic residuals were found as a function of magnitude. A linear fit to the proper motion data gives us:

$$
\begin{aligned}
& \mu_{\alpha} \cos \delta=0.187( \pm 0.133) \\
& \quad+0.982( \pm 0.009) \cdot\left(\mu_{\alpha} \cos \delta\right)_{\mathrm{Tyc} 2} ; \quad r=0.988 \\
& \begin{aligned}
& \mu_{\delta}= 0.201( \pm 0.132) \\
& \quad+1.016( \pm 0.008) \cdot\left(\mu_{\delta}\right)_{\mathrm{Tyc} 2} ; \quad r=0.992
\end{aligned}
\end{aligned}
$$

being $r$ the correlation coeficient.

Only 8 stars were found in this region from the Hipparcos catalogue (ESA 1997). By comparing our absolute proper motions with these common stars, the mean differences are (in the sense ours minus Hipparcos) -0.610 $(\sigma=1.710)$ and $-0.198(\sigma=1.658)$ in $\mu_{\alpha} \cos \delta$ and $\mu_{\delta}$,
Table 2. Mean precisions of proper motions as a function of the number of plates in the NGC 2548 region. (Units are mas $\mathrm{yr}^{-1}$.) $N$ is the number of stars.

\begin{tabular}{ccccc}
\hline \hline plates & $N$ & $\epsilon_{\mu_{\alpha} \cos \delta}$ & $\epsilon_{\mu_{\delta}}$ & $\epsilon_{\mu}$ \\
\hline 3 & 4 & 5.29 & 2.90 & 6.30 \\
4 & 8 & 2.92 & 3.12 & 4.44 \\
5 & 46 & 1.76 & 1.11 & 2.19 \\
6 & 119 & 1.19 & 0.89 & 1.53 \\
7 & 27 & 1.15 & 0.76 & 1.42 \\
8 & 20 & 0.90 & 0.70 & 1.17 \\
9 & 50 & 0.74 & 0.55 & 0.94 \\
10 & 199 & 0.59 & 0.46 & 0.77 \\
\hline \hline
\end{tabular}

respectively. We obtain the following linear fit:

$$
\begin{aligned}
& \mu_{\alpha} \cos \delta=-0.458( \pm 0.756) \\
& +1.005( \pm 0.013) \cdot\left(\mu_{\alpha} \cos \delta\right)_{\mathrm{HIP}} ; \quad r=0.999 \\
& \mu_{\delta}=-0.470( \pm 0.694) \\
& +1.011( \pm 0.014) \cdot\left(\mu_{\delta}\right)_{\mathrm{HIP}} ; \quad r=0.999 .
\end{aligned}
$$

\section{Membership determination}

Accurate membership determination is essential for further astrophysical studies of clusters. The fundamental mathematical model set up by Vasilevskis et al. (1958) and the technique based upon the maximum likelihood principle developed by Sanders (1971) have since been continuously refined.

An improved method for membership determination of stellar clusters based on proper motions with different observed precisions was developed by Stetson (1980) and Zhao \& He (1990). Zhao \& Shao (1994) then added the 


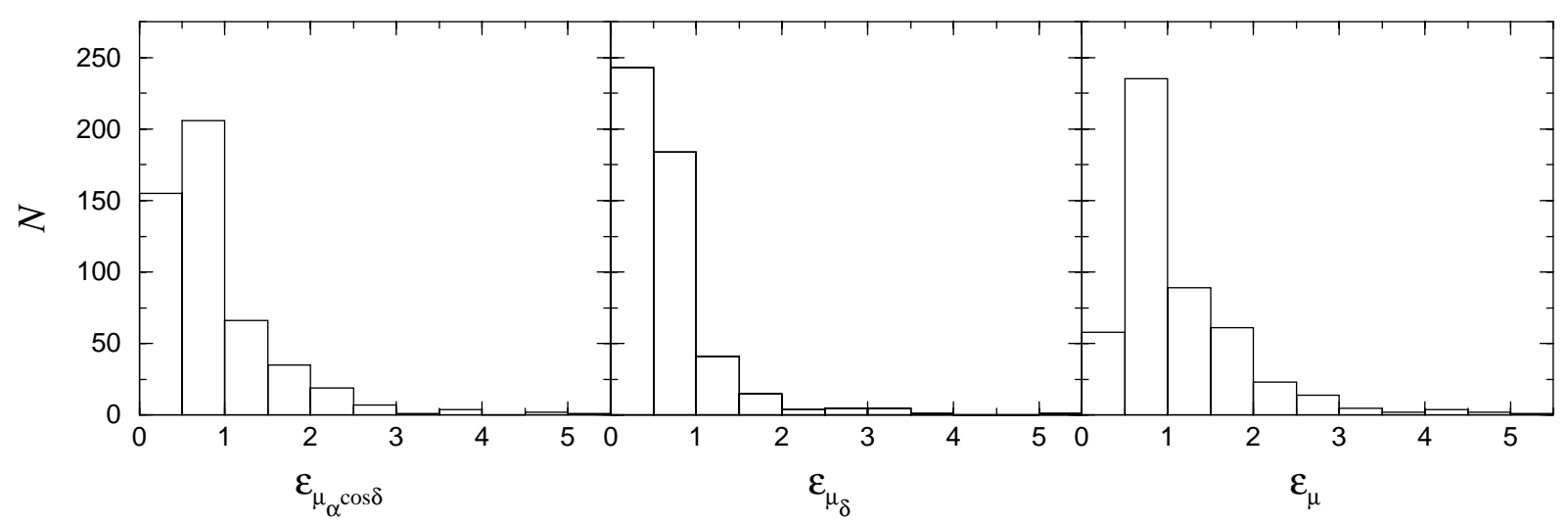

Fig. 2. The number of stars vs. the rms errors on proper motions in $\operatorname{mas} \mathrm{yr}^{-1}$.
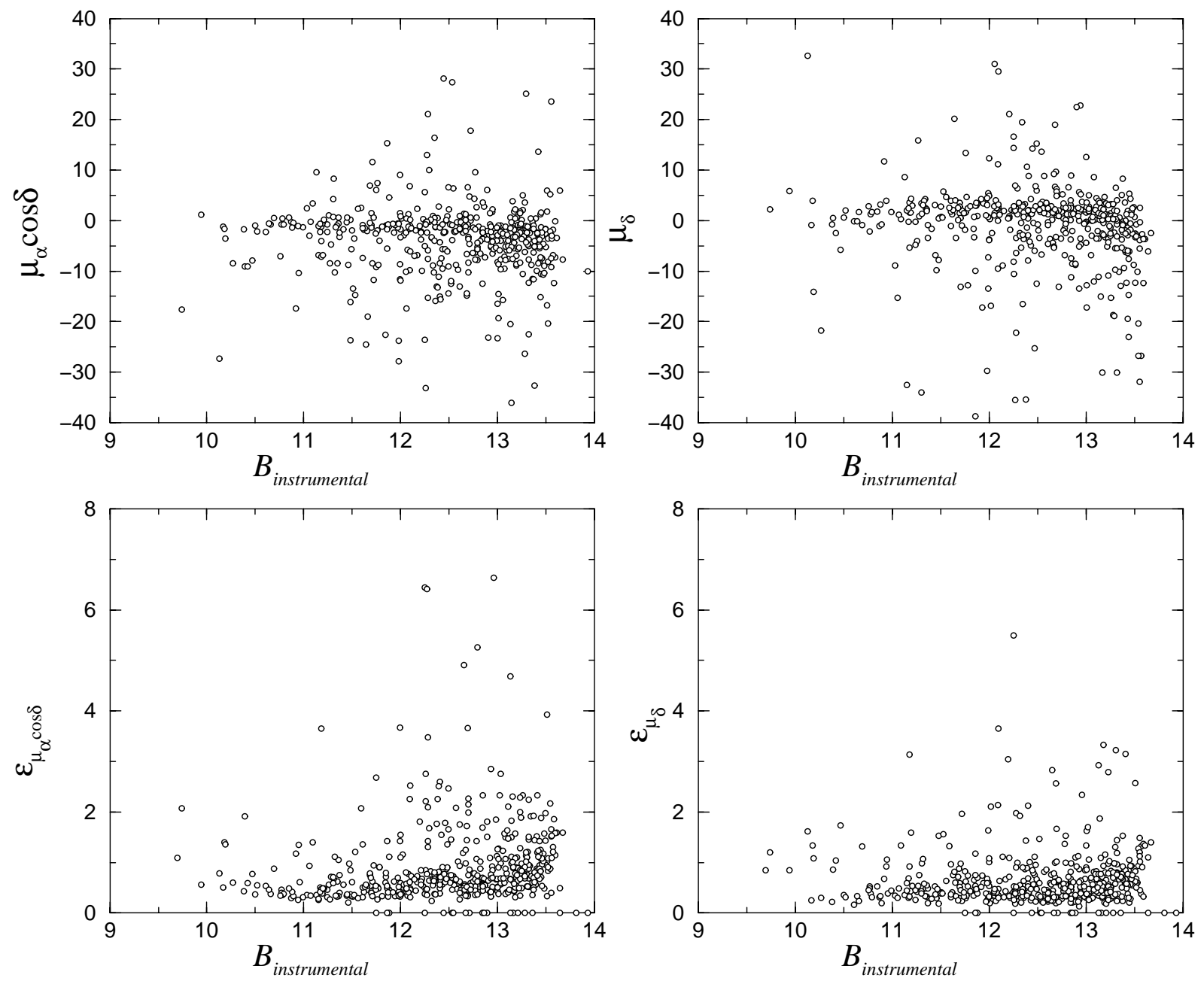

Fig. 3. Proper motions (top) and their errors (bottom) vs. instrumental magnitude. Null errors are from proper motions calculated with only two plates. (Units are mas $\mathrm{yr}^{-1}$ ).

correlation coefficient of the field star distribution to the set of parameters describing their distribution on the sky. This model has been frequently used (Wang et al. 1995, 1996, 2000).

We used a maximum likelihood method with a 9-parametric Gaussian model for the frequency function, as follows:

$\Phi=\Phi_{\mathrm{c}}+\Phi_{\mathrm{f}}=n_{\mathrm{c}} \cdot \phi_{\mathrm{c}}+n_{\mathrm{f}} \cdot \phi_{\mathrm{f}}$

where $\phi_{\mathrm{c}}$, and $\phi_{\mathrm{f}}$, are the probability density functions of cluster members and field stars respectively in the proper motion space, with $n_{\mathrm{c}}$ the normalized number of cluster stars, and $n_{\mathrm{f}}$ the normalized number of field stars. 

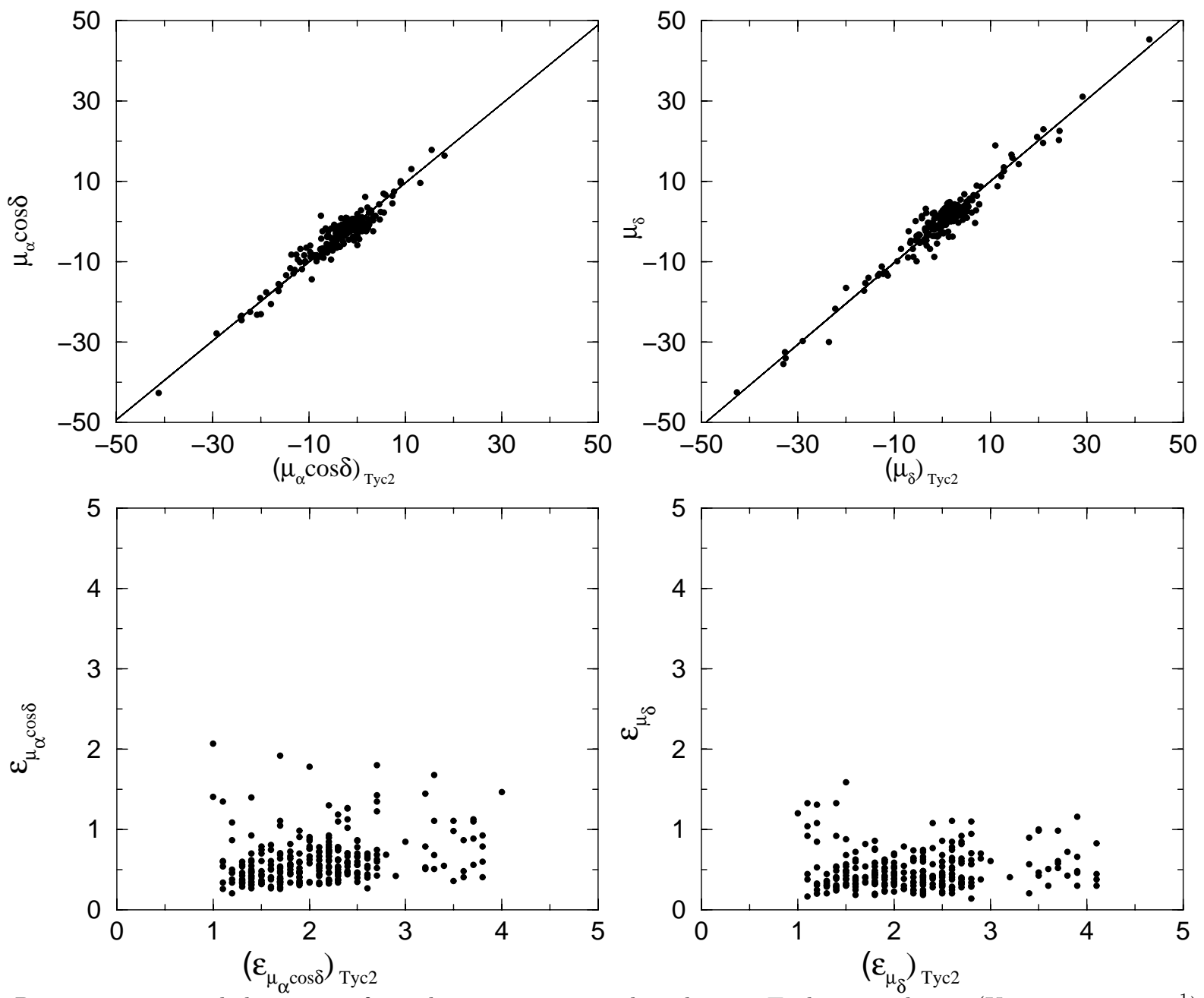

Fig. 4. Proper motions and their errors from this paper compared to those in Tycho-2 catalogue. (Units are mas yr ${ }^{-1}$ ).

The probability density function for the $i$ th star of the cluster can be written as follows:

$$
\begin{aligned}
\phi_{\mathrm{c}}(i)= & \frac{1}{2 \pi\left(\sigma_{\mathrm{c}}^{2}+\epsilon_{\left(\mu_{\alpha} \cos \delta\right)_{i}}^{2}\right)^{1 / 2}\left(\sigma_{\mathrm{c}}^{2}+\epsilon_{\left(\mu_{\delta}\right)_{i}}^{2}\right)^{1 / 2}} \\
& \times \exp \left\{-\frac{1}{2}\left[\frac{\left[\left(\mu_{\alpha} \cos \delta\right)_{i}-\left(\mu_{\alpha} \cos \delta\right)_{\mathrm{c}}\right]^{2}}{\sigma_{\mathrm{c}}^{2}+\epsilon_{\left(\mu_{\alpha} \cos \delta\right)_{i}}^{2}}\right.\right. \\
& \left.\left.+\frac{\left[\left(\mu_{\delta}\right)_{i}-\left(\mu_{\delta}\right)_{\mathrm{c}}\right]^{2}}{\sigma_{\mathrm{c}}^{2}+\epsilon_{\left(\mu_{\delta}\right)_{i}}^{2}}\right]\right\},
\end{aligned}
$$

where $\left[\left(\mu_{\alpha} \cos \delta\right)_{i},\left(\mu_{\delta}\right)_{i}\right]$ are the proper motions of the $i$ th star, $\left[\left(\mu_{\alpha} \cos \delta\right)_{\mathrm{c}},\left(\mu_{\delta}\right)_{\mathrm{c}}\right]$ the cluster mean proper motion, $\sigma_{\mathrm{c}}$ the intrinsic proper motion dispersions of member stars and $\left[\epsilon_{\left(\mu_{\alpha} \cos \delta\right)_{i}}, \epsilon_{\left(\mu_{\delta}\right)_{i}}\right]$ the observed errors on the proper motion components of the $i$ th star.

Analogously, for the field we have:

$$
\begin{aligned}
& \phi_{\mathrm{f}}(i)=\frac{1}{2 \pi\left(1-\gamma^{2}\right)^{1 / 2}\left(\sigma_{\left(\mu_{\alpha} \cos \delta\right)_{\mathrm{f}}}^{2}+\epsilon_{\left(\mu_{\alpha} \cos \delta\right)_{i}}^{2}\right)^{1 / 2}} \\
& \times \frac{1}{\left(\sigma_{\left(\mu_{\delta}\right)_{\mathrm{f}}}^{2}+\epsilon_{\left(\mu_{\delta}\right)_{i}}^{2}\right)^{1 / 2}} \exp \left\{-\frac{1}{2\left(1-\gamma^{2}\right)}\left[\frac{\left[\left(\mu_{\alpha} \cos \delta\right)_{i}-\left(\mu_{\alpha} \cos \delta\right)_{\mathrm{f}}\right]^{2}}{\sigma_{\left(\mu_{\alpha} \cos \delta\right)_{\mathrm{f}}}^{2}+\epsilon_{\left(\mu_{\alpha} \cos \delta\right)_{i}}^{2}}\right.\right. \\
& -\frac{2 \gamma\left[\left(\mu_{\alpha} \cos \delta\right)_{i}-\left(\mu_{\alpha} \cos \delta\right)_{\mathrm{f}}\right]\left[\left(\mu_{\delta}\right)_{i}-\left(\mu_{\delta}\right)_{\mathrm{f}}\right]}{\left[\sigma_{\left(\mu_{\alpha} \cos \delta\right)_{\mathrm{f}}}^{2}+\epsilon_{\left.\left(\mu_{\alpha} \cos \delta\right)_{i}^{2}\right]}^{1 / 2}\left[\sigma_{\left(\mu_{\delta}\right)_{\mathrm{f}}}^{2}+\epsilon_{\left(\mu_{\delta}\right)_{i}}^{2}\right]^{1 / 2}\right.} \\
& \left.\left.+\frac{\left[\left(\mu_{\delta}\right)_{i}-\left(\mu_{\delta}\right)_{\mathrm{f}}\right]^{2}}{\sigma_{\left(\mu_{\delta}\right)_{\mathrm{f}}}^{2}+\epsilon_{\left(\mu_{\delta}\right)_{i}}^{2}}\right]\right\},
\end{aligned}
$$

where $\left[\left(\mu_{\alpha} \cos \delta\right)_{\mathrm{f}},\left(\mu_{\delta}\right)_{\mathrm{f}}\right]$ are the field mean proper motion, $\left[\sigma_{\left(\mu_{\alpha} \cos \delta\right)_{\mathrm{f}}}, \sigma_{\left(\mu_{\delta}\right)_{\mathrm{f}}}\right]$ the field intrinsic proper motion dispersions and $\gamma$ is the correlation coefficient.

\section{Results and discussion}

The unknown parameters for the assumed $\Phi$ distribution are $\left[n_{\mathrm{c}},\left(\mu_{\alpha} \cos \delta\right)_{\mathrm{c}},\left(\mu_{\delta}\right)_{\mathrm{c}}, \sigma_{\mathrm{c}}\right]$ for the cluster and $\left[\left(\mu_{\alpha} \cos \delta\right)_{\mathrm{f}},\left(\mu_{\delta}\right)_{\mathrm{f}}, \sigma_{\left(\mu_{\alpha} \cos \delta\right)_{\mathrm{f}}}, \sigma_{\left(\mu_{\delta}\right)_{\mathrm{f}}}, \gamma\right]$ for the field population. Membership probability of the $i$ th star belonging to the cluster can be calculated from

$P_{\mathrm{c}}(i)=\frac{\Phi_{\mathrm{c}}(i)}{\Phi(i)}$.

By applying the standard maximum likelihood method, we obtained the 9 distribution parameters and their corresponding uncertainties, shown in Table 3, where the units of the proper motions and proper motion dispersions are mas $\mathrm{yr}^{-1}$. The quality of the fit should be optimized near the proper motion region occupied by the cluster stars, where the model is most crucial to providing reliable membership determination. Outliers cause the estimated distribution of field stars to be flatter than the actual one, thus increasing the final probability of 
Table 3. Distribution parameters and their uncertainties for NGC 2548. The units of $\mu$ and $\sigma$ are $\operatorname{mas}^{\mathrm{yr}^{-1}}$.

\begin{tabular}{lccccccc}
\hline & $n_{\mathrm{c}}$ & $\mu_{\alpha} \cos \delta$ & $\mu_{\delta}$ & $\sigma_{\mathrm{c}}$ & $\sigma_{\mu_{\alpha} \cos \delta}$ & $\sigma_{\mu_{\delta}}$ & $\gamma$ \\
\hline NGC 2548 & 0.382 & -1.41 & 1.64 & 1.23 & & & \\
& \pm 0.025 & \pm 0.12 & \pm 0.12 & \pm 0.08 & & & \\
field & & -4.89 & -1.63 & & 7.37 & 8.21 & -0.28 \\
& & \pm 0.07 & \pm 0.57 & & \pm 0.04 & \pm 0.29 & 0.03 \\
\hline
\end{tabular}

membership (Kozhurina-Platais et al. 1995; Cabrera-Caño \& Alfaro 1985; Zhao \& Tian 1985; Zhao et al. 1982). To minimize the effect of high proper-motion field stars in the model, we restricted the membership determination to the range $|\mu|<30$ mas $\mathrm{yr}^{-1}$.

Table 5 lists the results for all 501 stars in the region of the open cluster: Col. 1 is the ordinal star number; Cols. 2 and 3 are $\alpha_{\mathrm{J} 2000}$ and $\delta_{\mathrm{J} 2000}$; Cols. 4 and 6 are the respective absolute proper motions $\left(\mu_{\alpha} \cos \delta, \mu_{\delta}\right)$; Cols. 5 and 7 are the standard errors of the proper motions; Col. 8 is the membership probability of stars belonging to NGC $2548\left(P_{\mathrm{c}}\right)$; Col. 9 is the instrumental magnitude given by SExtractor; Col. 10 gives the number of plates used and Col. 11 the identification number in Tycho-2 for the stars in common.

The cross-identification of our 8 common stars with the Hipparcos catalogue is shown in Table 4. Comparison with the membership determination calculated by Baumgardt et al. (2000) for the seven stars in common shows complete agreement.

Figures 5 and 6 show the proper motion vector-point diagram and the position distribution on the sky for all the measured stars respectively, where "०" denotes a member of NGC 2548 with $P_{\mathrm{c}} \geq 0.7$, and all other stars are considered field stars indicated by "+".

The cluster membership probability histogram (Fig. 7) shows a very clear separation between cluster members and field stars. The number of stars with membership probabilities higher than 0.7 for NGC 2548 is 165 . The average cluster membership probability is 0.90 , giving a contamination by field stars not larger than $10 \%$. If these stars were considered as belonging to the field, the apparent deficiency of field stars in the central region would vanish. Likewise, the contamination by cluster stars in the field is not larger than $6 \%$.

The present results for this cluster should be complemented with photometry and radial velocity studies. To this end, our group is working on a complete photometric study of the region.

\subsection{Effectiveness of membership determination}

Contamination by background and foreground objects due to the observational projection effect cannot be avoided. Following Shao \& Zhao (1996), we can assess the effectiveness of our membership determination, which is set as:

$E=1-\frac{N \sum_{i=1}^{N}\left\{P_{\mathrm{c}}(i)\left[1-P_{\mathrm{c}}(i)\right]\right\}}{\sum_{i=1}^{N} P_{\mathrm{c}}(i) \sum_{i=1}^{N}\left[1-P_{\mathrm{c}}(i)\right]}$, where the greater $E$, the more effective the membership determination.

We thus obtained an effectiveness of membership determination of 0.77 for NGC 2548. Figure 3 of Shao \& Zhao (1996) paper shows that the effectiveness of membership determination of 43 open clusters ranges from 0.20 to 0.90 and the peak value is 0.55 . Compared with previous studies (Shao \& Zhao 1996; Tian et al. 1998; Balaguer-Núñez et al. 1998), the effectiveness of membership determination for this open cluster is significantly high.

\subsection{Space velocity and galactic orbit}

By combining our absolute proper motion results with radial velocity (Piatti et al. 1995), and the age and distance of the cluster from the Catalogue of Lyngå (1987), we determined the space velocity and the galactic orbit of NGC 2548.

To obtain the velocity of the cluster in the galactocentric frame, we assumed the motion of the Sun in the LSR to be $(U, V, W)_{\odot}=(9.7,5.2,6.7) \mathrm{kms}^{-1}$ (Bienaymé 1999). We adopted the current IAU standard values of $V_{\mathrm{LSR}}=220 \mathrm{kms}^{-1}$ for the local circular rotation velocity and $R_{\odot}=8.5 \mathrm{kpc}$ for the Sun galactocentric distance. The resulting galactocentric position and velocity of the cluster are $(x, y, z)=(-8.89,-0.44,+0.16) \mathrm{kpc}$ and $(U, V, W)=(0,222,7) \mathrm{km} \mathrm{s}^{-1}$. These vectors, together with the galactic gravitational potential model of Allen \& Santillan (1991) with the estimated cluster age of $\log t=8.5$, determine the orbit of the cluster in the Galaxy. The resulting orbit is characterized by an eccentricity of 0.022 , radial oscillations between distances from the galactic center of 8.67 and $9.04 \mathrm{kpc}$ and vertical oscillations between -0.19 and $0.19 \mathrm{kpc}$. The cluster has made 1.18 revolutions around the galactic center and 8 crossings of the galactic disk in its lifetime. Assuming the distance of Clariá (1985) does not affect the results. We currently observe the cluster near its maximum distance from the plane. This is consistent with the expectation of a higher probability of finding the cluster near its maximum $|z|$, where the cluster will spend more time due to its vertical motion.

\section{Summary}

Proper motions and their corresponding errors of 501 stars within a $1.6 \times 1.6$ area in the NGC 2548 region were determined from automatic MAMA measurements of 


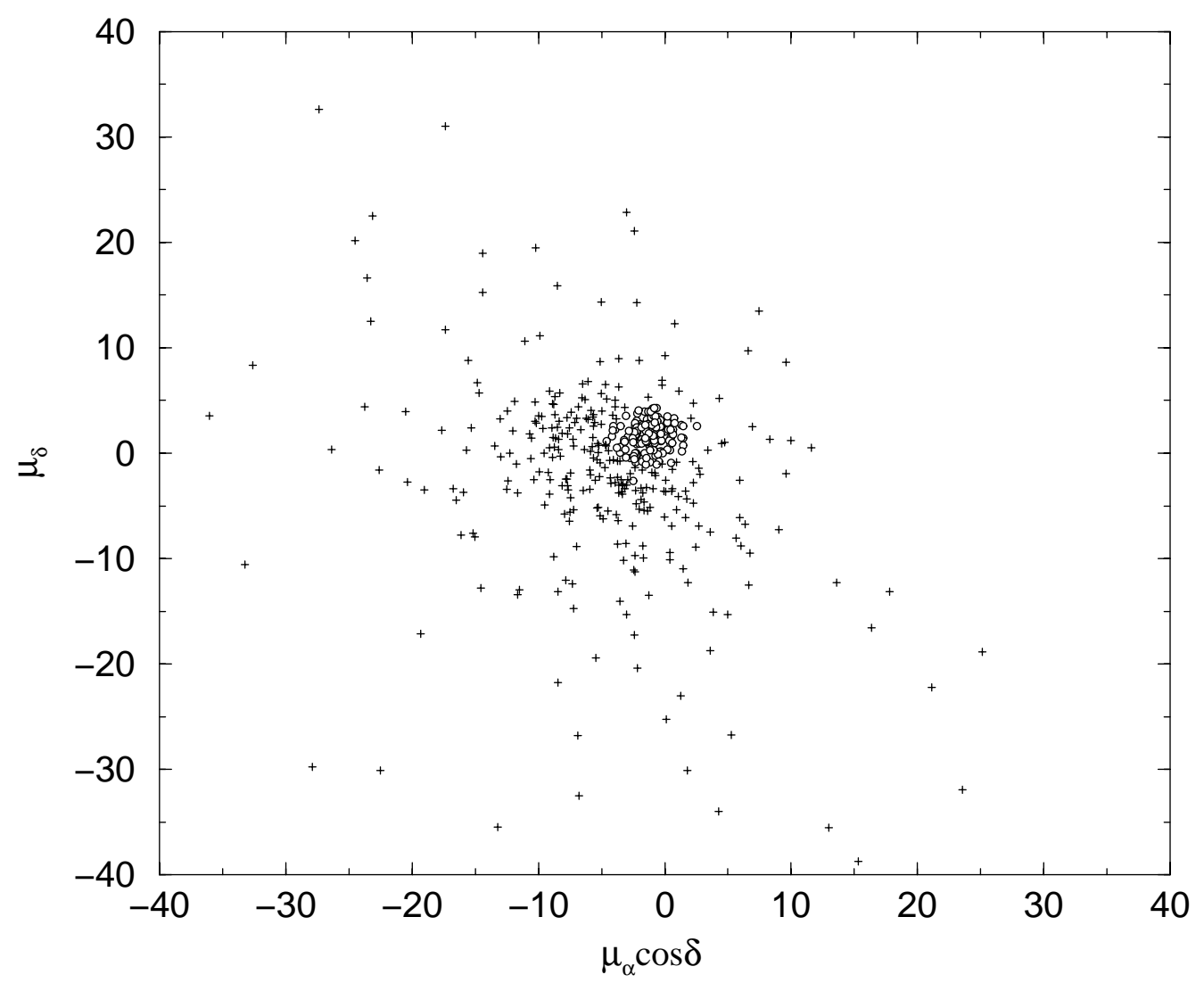

Fig. 5. The proper motion vector-point diagram of NGC 2548. (Units are mas $\mathrm{yr}^{-1}$.) ("o" denotes a member of NGC 2548 with $P_{\mathrm{c}} \geq 0.7$, " +" a field star with $\left.P_{\mathrm{c}}<0.7\right)$.

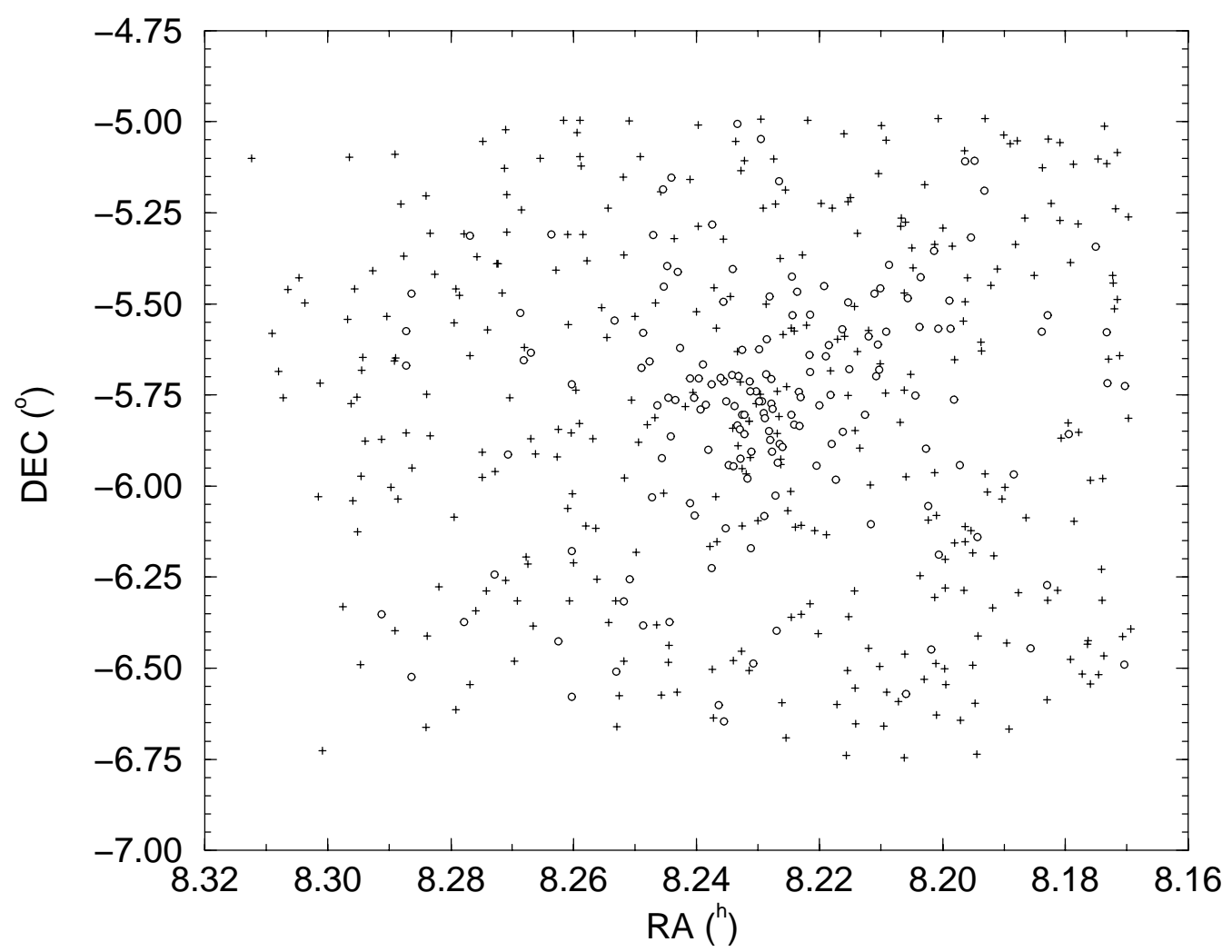

Fig. 6. The position distribution of stars in NGC 2548 area. ("o" denotes a member of NGC 2548 with $P_{\mathrm{c}} \geq 0.7$, "+" a field star with $\left.P_{\mathrm{c}}<0.7\right)$. 
Table 4. The cross-identification of stars in common with the Hipparcos catalogue and the membership determination by Baumgardt et al. (2000). Comparison with the results from this paper $\left(P_{\mathrm{c}}\right)$ gives complete agreement.

\begin{tabular}{cccccc}
\hline Table 5 & Hipparcos & Tycho-2 & BDA & Member & $P_{\mathrm{c}}$ \\
\hline 257 & 40110 & $4859 \_00078 \_1$ & 366 & $\mathrm{~N}$ & 0.00 \\
140 & 40238 & $4859 \_00250 \_1$ & 1005 & $\mathrm{M}$ & 0.87 \\
139 & 40254 & $4859 \_00036 \_1$ & 1073 & $\mathrm{M}$ & 0.77 \\
336 & 40281 & $4859 \_00921 \_1$ & & & 0.00 \\
133 & 40302 & $4855 \_01706 \_1$ & 1320 & $\mathrm{M}$ & 0.78 \\
234 & 40348 & $4859 \_01156 \_1$ & 1560 & $\mathrm{M}$ & 0.88 \\
162 & 40362 & $4859 \_00092 \_1$ & 1628 & $\mathrm{M}$ & 0.84 \\
42 & 40498 & $4856 \_00072 \_1$ & 2184 & $\mathrm{~N}$ & 0.00 \\
\hline
\end{tabular}

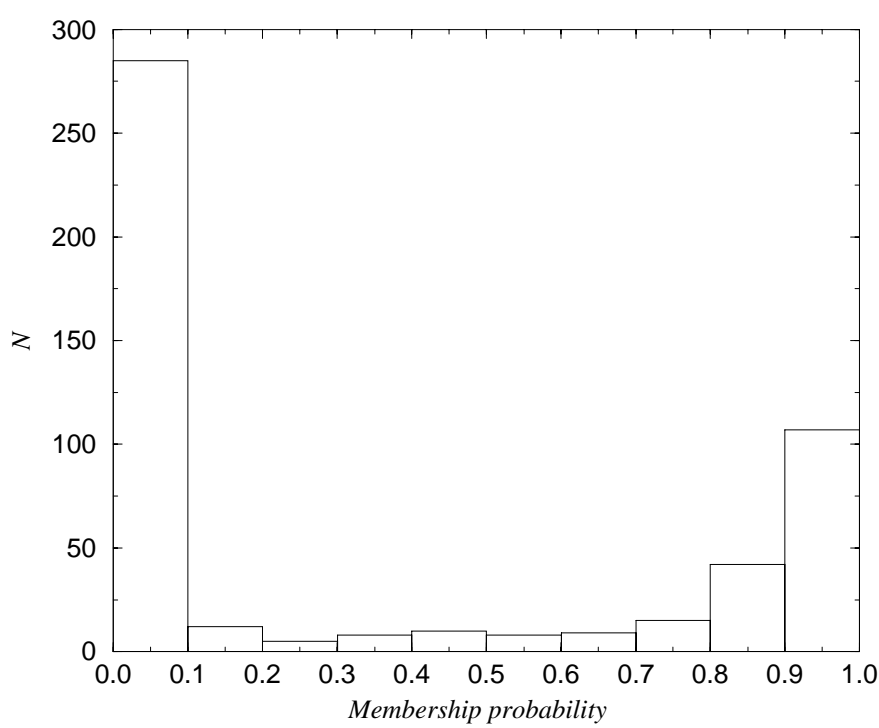

Fig. 7. The histogram of cluster membership probability of NGC 2548.

10 plates, five of which were newly taken. Comparison with Tycho-2 and Hipparcos Catalogues shows good agreement and underlines the precision of the proper motions derived in this paper. These proper motions are then used to determine membership probabilities of the stars in the region. We obtained 165 stars with membership probabilities higher than 0.7 .

Acknowledgements. We would like to thank Prof. J. J. Wang for useful discussions and Dr. D. Galadí-Enríquez for help and useful comments. We would also like to thank R. Chesnel and P. Toupet for scanning and prereduction of the plates. This study was partially supported by the National Natural Science Foundation of China Grant No. 19733001 and by Joint Laboratory for Optional Astronomy of CAS. L.B-N. gratefully acknowledges financial support from Wang Kuan Cheng Fund, Chinese Academy of Sciences. This study was also partially supported by the contract No. A\&A2000-0937 with MCYT.

\section{References}

Allen, C., \& Santillan, A. 1991, Rev. Mex. Astron. Astrofis., 22,255
Balaguer-Núñez, L., Tian, K. P., \& Zhao, J. L. 1998, A\&AS, 133,387

Baumgardt, H., Dettbarn, C., \& Wielen, R. 2000, A\&AS, 146, 251

Bertin, E., \& Arnouts, S. 1996, A\&AS, 117, 393

Bienaymé, O. 1999, A\&A, 341, 86

Cabrera-Caño, J., \& Alfaro, E. J. 1985, A\&A, 150, 298

Clariá, J. J. 1985, A\&AS, 59, 195

Collinder, P. 1931, Lund Observatory Annals, No. 2

Ebbighausen, E. G. 1939, ApJ, 90, 689

Eichhorn, H., \& Williams, C. A. 1963, AJ, 68, 221

ESA, 1997, The Hipparcos and Tycho Catalogues, SP-1200

Guibert, J., Soubiran, C., \& Geffert, M. 1990, in Colloque André Danjon, ed. N. Capitaine, \& S. Débarbat (Paris), 211

Høg, E., Fabricius, C., Makarov, V. V., et al. 2000, A\&A, 357, 367

Kozhurina-Platais, V., Girard, T. M., Platais, I., et al. 1995, AJ, 109, 672

Li, H. 1954, Ann. Zô-Sè Obs., 23, 1

Lyngå, G. 1987, Catalogue of Open Cluster, Centre de Données Stellaires, Strasbourg

Messier, C. 1850, CDT, 1784, 227

Pesch, P. 1961, ApJ, 134, 602

Piatti, A. E., Clariá, J. J., \& Abadi, M.G. 1995, AJ, 110, 2813

Russel, J. L. 1976, Ph.D. Thesis, University of Pittsburgh

Sanders, W. L. 1971, A\&A, 14, 226

Shao, Z. Y., \& Zhao, J. L. 1996, Acta Astron. Sin., 37, 377

Soubiran, C. 1992, A\&A, 259, 394

Stetson, P. B. 1980, AJ, 85, 387

Tian, K. P., Zhao, J. L., Shao, Z. Y., \& Stetson, P. B. 1998, A\&AS, 131, 89

Trumpler, R. J. 1930, Lick Obs. Bull., 14, 154

Twarog, B. A., Ashman, K. M., \& Anthony-Twarog, B. J. 1997, AJ, 114, 2556

Vasilevskis, S., Klemola, A., \& Preston, G. 1958, AJ, 63, 387

Wang, J. J., Zhu, G. L. \& Wang, X. M. 1982, Ann. Shanghai Obs., 4, 92

Wang, J. J., Chen, L., Zhao, J. H., \& Jiang, P. F. 1995, A\&AS, 113,419

Wang, J. J., Chen, L., Zhao, J. H, \& Jiang, P. F. 1996, CA\&A, 20,364

Wang, J. J., Chen, L., Wu, Z. Y., et al. 2000, A\&AS, 142, 373

Zhao, J. L., \& He, Y. P. 1990, A\&A, 237, 54

Zhao, J. L., \& Shao, Z. Y. 1994, A\&A, 288, 89

Zhao, J. L., \& Tian, K. P. 1985, AcASn, 26, 152

Zhao, J. L., Tian, K. P., Xu, Z., \& Yin, M. 1982, ChA\&A, 6, 344 